\title{
La teoría crítica: de Frankfurt a Habermas Una "traducción" de la teoría de la acción comunicativa a la sociología
}

José Antonio Noguera

Universitat Autònoma de Barcelona. Departament de Sociologia

08193 Bellaterra (Barcelona). Spain

\section{Resumen}

Este artículo pretende ser una primera introducción para no iniciados a la teoria sociológica de Jürgen Habermas. Se analiza la teoría de la acción comunicativa de Habermas desde el "giro linguístico" hasta la teoría de la sociedad pasando por la teoría de la acción. Se argumenca que la teoría de Habermas parte de un concepto de racionalidad comunicativa flosoficamente fundamentado y no dogmático, alternativo a la racionalidad instrumental-estratégica; que su teoría de la acción comunicativa provee de un modelo de acción alternativo al de las teorlas de la elección racional; que su teoría de la sociedad articulada en dos niveles - sisterna y mundo de la vida - supera las antinomias tanto del funcionalismo como de la fenomenología, conectando con la tradición marxista; y, por último, que todo ello conserva una conexión entre teoría y práctica típica de cualquier teoría crítica de la sociedad.

Palabras clave: teoría crítica, reoría social, Habermas, giro linguístico, acción comunicativa, sistema, mundo de la vida.

Abstract. Critical theory: from Frankfurt to Habermas. A utranslationn of the theory of communicative action to sociology

This article seeks to be a first introduction for those not iniciated in the sociological theory of Jürgen Habermas. It analyzes Habermas' theory of communicative action from the "linguistic turn" to the theory of society going through the theory of action. It is argumented that Habermas' theory starts from a concept of communicarive rationality which is philosophically grounded and not dogmatic, as an alternative to instrumental-strategic rationality; that his theory of communicative action gives a model of action, alternative to that of the rational choice theories; that his two-level theory of society avoids the theoretical problems of both functionalism and phenomenology, connecting with the marxist tradition; and to finish with, that everything preserves a connection between theory and practice which is typical of any critical theory of society.

Key words: critical theory, social theory, Habermas, linguistic turn, communicative action, system, lifeworld. 


\section{Sumario}

Introducción a Habermas

El "giro linguístico" y el "giro pragmático"

La teoría de la acción de Habermas: acción comunicativa y acción estratégica
La teoría de la sociedad de Habermas: sistema y mundo de la vida

A modo de conclusión: teoría y práctica en Habermas

Bibliografía

\section{Introducción a Habermas}

De entre todos los herederos de la tradición de la Escuela de Frankfurt ${ }^{l}$, es Jürgen Habermas quien ha desarrollado una propuesta teótica más acabada, hasta el punto de haber transformado por completo la faz de la teoría crítica original. En su ya larga y prolija trayectoria intelectual, Habermas ha atravesado diversas etapas y ha tratado prácticamente todos los ternas y discusiones de la teoría social y la filosofía contemporáneas. Se trata, sin duda, de uno de los principales teóricos sociales de nuestros días ${ }^{2}$.

Habermas, piensan muchos, es algo así como "un Parsons de izquierdas", lo que C. W. Mills habría llamado peyorativamente un "tebrico supremo", y ello provoca tanto rechazos viscerales en unos como exageradas devociones en otros. Hay algo de cierto en tal calificativo, no hay duda. Aún así -e independientermente de la deliberada exageración de Mills respecto a ParsonsHabermas ofrece, al mismo tiempo, muchas más cosas que atenúan el carácter superabstracto de gran parte de su obra: su planteamiento ha sido siempre histórico, y conectado con las preocupaciones políticas de la izquierda de la época; ha intentado fundamentar sus teorfas en base a la evidencia empírica disponible; ha tomado en consideración y discutido con casi todas las dernás escuelas de teoría sociológica y filosofía; ha intervenido públicamente en numerosos debates directamente políticos, comprometiéndose con posiciones muchas veces polémicas; se ha sometido y ha respondido a numerosos exámenes críticos por parte de todo tipo de autores, a partir de los cuales ha revisado y ha

1. Sobre la Escuela de Frankfurr y la primera generación de la teoría crírica, ver Jay (1973), Honnerh (1987) y Held (1980). Más bibliografia en castellano puede encontrarse referenciada en Mardones (1990). El estudio más actualizado, detallado $y$ completo de que se dispone hoy sobre la Escuela de Frankfurt es sin duda el de Wiggershaus (1986).

2. La bibliografia existente sobre Habermas es ya a todas luces inabarcable. De todas maneras, pueden encontrarse buenas introducciones a su obra en Bernstein (1985), Giddens (1985), Mardones (1985) o jiménez Redondo y orros (1988). Algunos estudios clásicos sobre Habermas, como el de McCarthy (1978), tienen el inconventente de haber quedado ya superados por el frenético ritmo de trabajo del alemán (aunque en este caso es también útil el epílogo sobre la Teoría de la acción comunicativa afiadido a la edición española de 1987). En inglés, introducciones más breves, pero más actualizadas, son las de Horster (1992) y Pusey (1987). Esrudios más detallados sobre sus aportaciones recientes pueden hallarse, también en inglés, en White (1988 y 1995), Holub (1991), Rasmussen (1990), Outhwaite (1994) y Bernstein (1995). 
corregido sus ideas ${ }^{3}$; ha depurado la teoría crítica de sus tentaciones más elitistas y oscurantistas, abandonando la dialéctica, la "crítica negativa", el psicoanálisis, la filosofía de la historia y cualquier deriva "utopista". Evidentemente, cormo ocurre con cualquier empresa de similar envergadura, en la de Habermas existen no pocos puntos oscuros. Pero aquí interesa señalar que se trata, hoy por hoy, con todas las críticas y matizaciones que se quiera, de la alternativa más rigurosa, elaborada y coherente para quienes no renuncien a una comprensión global de las sociedades capitalistas occidentales desde una racionalidad que no se reduzca en términos estratégicos - como hacen las teorías de la elección racional - ni sistémicos - como ocurre con el neofuncionalismo-, y desde unos valores e intenciones que entroncan con el proyecto de ciencia social crítica que inició Marx 4 .

Es cierto también que la teoría de Habermas supone, respecto del proyecto original de la teoría crítica, una concentración en los esfuerzos propiamente teóricos y una considerable moderación de sus objetivos políticos. Se podría decir que la teoría crírica, con Habermas, se «institucionaliza» en los centros académicos oficiales y se "socialdemocratiza" políticamente, aunque sin duda quedarse ahí sería una simplificación excesiva. Y es que Habermas desarrolla su pensamiento en un contexto histórico y político muy distinto del que vio nacer a la Escuela de Frankfurt: la Alemania ureconstruida" de la posguerra, el desarrollo y posterior crisis de los estados de bienestar, la progresiva desradicalización del movimiento obrero, el surgimiento de nuevas desigualdades y nuevos movimientos sociales, en suma, la creciente complejidad de las sociedades capitalistas occidentales, es de esperar que den lugar a una manera diferente de teorizar.

Lo que se intentará, en lo que sigue, es ofrecer una idea general --sin duda selectiva y discutible - de los derroteros por los que hoy camina la teoría crítica en la obra de Habermas. A riesgo de parecer apologético, dejaré para ocasión más propicia una valoración crítica de la teoria de Habermas, así como una revisión de otras aportaciones menos conocidas que la suya a la teoría crítica actual. La segunda advertencia es que, para no complicar excesivamente el análisis ni hacerlo demasiado repetitivo respecto de la numerosísima bibliografía ya existente, lo limitaré a:

3. Algunas de esas discusiones críticas han quedado recogidas, junto con las correspondientes réplicas por parte de Habermas, en volúmenes colectivos: ver Bernstein (ed.) (1985), Held y Thompson (eds.) (1982), Honneth y Joas (eds.) (1991), ० Calhoun (ed.) (1992).

4. A este respecto, y a pesar de que muchos marxistas "oficiales" abominan de su teoria, Habermas ha manifestado en ocasiones que se ve a si mismo como continuador del proyecto de Marx, e incluso que se siente "el último tnarxista" (Habermas y otros, 1992a: 469). Eilo es tanto más significativo si consideramos que en los años ochenta el marxismo y la teoría crítica fueron desplazados del centro de la atención teórica internacional por ocras corrientes como las ceorias de la elección racional o el postestruccuralismo y postmodernismo franceses; sobre este punto, ver Anderson (1983) y Kellner (1993: 45). 
a) las contribuciones de Habermas a partir de 1981, año de publicacion de la Teoria de la acción comunicativa (en adelante TAC);

b) los aspectos más sociológicos de tales aportaciones, prescindiendo, hasta donde sea posible, de los filosóficos ${ }^{6}$. Concluiremos con unas breves observaciones sobre cómo plantea Habermas la conexión entre teoría y práctica que caracteriza a toda teoría crítica.

5. La obra de Habermas, como se ha dicho, es muchisimo más extensa de lo que un simple artículo puede abarcar. Las siguientes líneas pueden servir de "guía" general de la misma.

En los años sesenta Habermas, todavía muy cercano a Adorno y Horkheimer, se dedicó a la crítica del positivismo desde una idea dialéctica de la ciencia (ver sus aportaciones al libro colectivo La disputa del positivismo en la sociologia alemana -Habermas, 1969-). En esa época publica también la que sus detractores suelen considerar su mejor obra, Historia y critica de la opinión pública (1962) — nefasta traducción al espafol del título original alemán, La transformación estructural de la "esfera pública)-, en la que analiza la degradación de la vida pública en las modernas democracias capitalistas desde un punto de vista aún bastante marxista (influido por su director de resis, el historiador del movimiento obrero Wolfgang Abendrorh). A finales de los años sesenta destacan su crítica de la tecnocracia y de los usos ideologicos de la ciencia en Ciencia y técnica como uideologtas (1968a), y su intento de fundamentar epistemologicamente la teotia crítica en Conocimiento e interés (1968b), obras ambas en las que ya se observa incipiente el llamado "giro lingüistico". A caballo entre esta década y la siguiente se encuentran también La lógica de las ciencias sociales (1977) y Teoria y praxis (1971), colecciones de artículos y estudios sobre metodologla, epistemologia de las ciencias sociales, $y$ filosofia politica.

Los años setenta verán avanzar la obra de Habermas en dos direcciones: por un lado, profundizará el "giro lingǘsticon mediante una serie de trabajos sobre teoría de la comunicación y de la acción social claramente preparatorios de la TAC - la mayoría de los cuales están recogidos en Teoria de la acción comunicativa: Complementos y estudios previos (1984b) - y que plantean por vez primera su epragmática universaln del lenguaje; por otro fado, y partiendo de esos puntos de vista, actualizará y transformará la teoria marxista para aplicarla al análisis del capitalismo contemporáneo en libros como Problemas de legitimación en el capitalismo tardio (1973) —orro de los más apreciados por los no habermasianos- y La reconstrucción del materialismo bistórico (1976), en el que además presenta su teoría de la evolución social y sus primeros estudios sobre el tema de la identidad colectiva.

En los afros ochenta, y como uno de los desarrollos de la TAC, Habermas abordará la construcción de una ética comunicativa: los articulos sobre este tema están agrupados en libros como Conciencia moral y acción comunicativa (1983) o Escritos sobre moralidad y eticidad (1984-1987). Al hito de esta ética, y de sus implicaciones para la teoría y la filosofia política, los últimos años ochenta y primeros noventa contienen también aportaciones sobre el tema de la identidad nacional y la ciudadanía (Habermas, 1988a y 1992a). Esta derivación hacia la filosofia política se ha convertido ya en la principal línea de trabajo de Habermas, cristalizada en una teoría de la democracia y del derecho, que, entre orras cosas, intenta terciar en la discusión filosófica acctal entre «liberales" y "comunitaristass: ver Habermas (1989, 1992b, 1992c y 1995); también, sobre este particular, Larmore (1994) y Velasco (1994).

6. En las más de mil páginas de la Teoria de la accíón comunicativa, resultado de unos diez años de trabajos, Habermas expone de forma global y sistemática sus teorías de la sociedad, de la racionalidad, de la acción y de la evolución social, además de discutir e integrar casi toda la tradición sociológica anterior, manxista o no (Weber, Durkheim, Mead, Schütz y Parsons son también centrales en su análisis). Sintetizar y utraduciro la TAC es tarea ardua, y sin embargo necesaria dada su enorme complejidad y su grado de abstracción. Por otro lado, en los quince años transcurridos desde su publicación, Habermas ha profundizado y revisado algunos de los principales aspectos de aquel libro — sobre todo en El discurso filosoffi- 


\section{El "giro lingüístico" y el "giro pragmático»}

La TAC no puede entenderse si no se hace referencia al cambio de paradigma que ha tenido lugar durante el presente siglo en la filosofía occidental. Si la filosofía clásica se basaba en el paradigma ontológico (que se pregunta por el ser), y la moderna en el mentalista (lo que Habermas ilama "filosofía de la conciencian, que se interesa por la posibilidad del conocimiento), en el siglo $\mathrm{XX}$-a partir de filosofos tan distintos como Heidegger, Wittgenstein, Humboldt, Frege y otros- se inaugura el paradigma lingüistico, que rompe con el esquema sujeto/objeto, considerando el lenguaje como eabridor de mundo" y como el medio y el «lugar» del pensamiento y la racionalidad humanas ${ }^{7}$.

Habermas es muy consciente de que hoy día no podemos ya caer por detrás de este "giro lingüísticon. Y lo que propone es una particular versión del mismo, que aplica a la teorfa social y a la sociolog ${ }^{8}{ }^{8}$. Acaso la mejor manera de introducirla sea partir de la crítica que, ya en los años sesenta, Habermas hizo a Marx y a sus supuestos antropológicos, y que básicamente ha seguido suscribiendo después.

Habermas piensa que el planteamiento de Marx padece de un "reduccionismo categorial" que debe ser corregido. Según su análisis, Marx fue presa, como en general todos los teóricos de su época, de las categorías de la ufilosofia de la conciencian, el paradigma filośbico dominante en la modernidad. Tal paradigma se basa en el modelo de un actor aislado que se enfrenta teleológicamente -es decir, buscando el éxito en la consecución de sus objetivos o finalidades- con un mundo externo "objetivo", ya sea éste social o natural. Es este modelo el que lleva a Marx, según Habermas, a conceprualizar la categoría de trabajo como la esencial para el proceso de hominización - por el cual la especie humana se diferencia del resto de especies animales-: el ser humano es, ante todo, homo faber, ser que se enfrenta al mundo y lo transforma instrumentalmente según sus deseos. Pero esta antropología filosófica, afirma Habermas, es reduccionista: deja de lado otra dimensión esencial para la constitución de la especie humana como tal, cual es la de la interacción social, y más concretamente la de la comunicación y el lenguaje. Tanto uno - trabajocomo otra - comunicación- son esenciales para la autorreproducción del ser

co de la Modernidady en Pensamiento postmetafisico-, con lo que la tarea se complica aún más. La exposición cronológica de tales desarrollos, aun siendo la opción más rigurosa académicamente, tendría el efecto de alargar en exceso este artículo y hacerlo repetitivo; por esta razón se ha optado por la ficción de presentar la teoría en su versión más actual, sin especificar los cambios de matiz que haya podido ir sufriendo en esos af́os. Por último, hay que decir que un intento de síntesis y "traducción de la TAC como éste - que quiera ser útil para "no iniciados" en su estudio- puede hacer perder ciertos detalles y surilezas que el fílósofo o el reórico socjal avezados echarán de menos. Confiamos, no obstante, en que la claridad no está reñida con el rigor, y en que ésta puede ser también una buena manera de dar sentido a un artículo sobre un tema ya muy trillado.

7. Ver Vilar (1993).

8. Ver los prolegómenos de este inzento en Habermas (1984b), cap. 1 : "Lecciones sobre una fundamentación de la sociología en términos de teoría del lenguajes. 
humano y de la sociedad'. Marx estaría inmerso en el que Habermas llama "paradigma productivista", que reduce toda la praxis social a trabajo social sin advertir que en las interacciones lingüísticas existen potenciales no reductibles sin más al tipo de actividad teleológica que domina en la esfera del trabajo. El paradigma lingǘstico marca el fin de este esquema categorial del que Marx fue partícipe.

Los seres humanos, viene a decir Habermas, no sólo se reproducen mediante actividades teleológicas orientadas a un fin, sino también mediante procesos cooperativos de interacción que les permiten establecer fines comunes y coordinarse para llevarlos a la práctica. El entendimiento lingüistico es, entonces, hecho fundante de toda sociedad. La conciencia individual no se forma en la relación del individuo con un "mundo exterior" - natural o social- que se le presenta como "objeton, sino a través de la intersubjetividad, de la interacción comunicativa con otros sujetos conscientes en el contexto institucional de una sociedad, contexto en el que los individuos se desenvuelven en actitud "participante" y no sólo "objetivantes" ${ }^{10}$.

Es necesaria, por tanto, una reconstrucción de la teoría social crítica que incorpore este tránsito de la "filosofía de la conciencia» y del "paradigma productivista" al paradigma comunicativo. Para Habermas no se trata de complementar un paradigma con el otro, sino de una sustitución en toda regla: el paradigma comunicativo es más amplio y más comprehensivo, y por tanto capaz de incluir y explicar el productivista (Habermas, 1986: 213), y ello por varias razones: el paradigma comunicativo a) se basa en un concepto de racionalidad que incluye los elementos de racionalidad instrumental propios del productivista, pero que va más allá para añadir las dimensiones práctico-morales y estético-expresivas; b) puede explicar adecuadamente las innovaciones evolutivas en el ámbito de las estructuras de comunicación e interacción social, y no sólo en el de las fuerzas productivas: esto es, puede entender el "progreso" - de forma no determinista ni unecesarian- como algo más que un dato técnico-productivo; c) puede fundamentar normativamente de forma no arbitraria ni dogmática la teoría crítica de la sociedad, problema que nunca supieron resolver Adorno, Horkheimer o Marcuse, y al que Marx simplemente no dio importancia; o, en otras palabras, puede dar razón de las implicaciones normativas de la teoría crítica; d) descubre que en la comunicación existen

9. Es en las estructuras del trabajo y del lenguaje donde se han producido los procesos que han llevado a la forma de reproducción especificamente humana de la vida y, con ello, a la situación de origen de la evolución social. Trabajo y lenguaje son más antiguos que el ser bumano y que la sociedads (Habermas, 1976: 138-139; s.o.).

10. La argumentación más reciente de Habermas sobre todo este tema puede estudiatse más en detalle en Habermas (1985a), cap. 11: "Otra manera de salir de la filosofía del sujero: razón comunicativa versus razón centrada en el sujeton; en este mismo libro, las páginas 79-90, 99-107, 375-381 y 403-422 clarifican lo que Habermas llama el ufin del paradigma productivistas, así como su crítica a Marx. Sobre la mutua dependencia entre individuación y socialización lingüística, ver también Habermas (1988b), cap. 8: "Individuación por via de socialización. Sobre la teoría de la subjetividad de G. H. Meadp. 
potenciales de emancipación social que van más allá de una hoy utópica "reconciliación del trabajador con su propio producto". Por todo ello, aftrma Habermas, "el modelo comunicativo es inclusivo" (ibídem).

Antes de continuar, es necesaria una advertencia: el cambio de paradigma que opera Habermas en la teoría crítica no puede entenderse en absoluto como un giro "idealista" de la misma, tal y como apresurada y prejuiciadamente han hecho algunos marxistas como Therborn (1971). Habermas no afirma de ningún modo que la sociedad pueda entenderse en términos exclusivamente comunicacionales o de interacción linguística, ni que la cultura y la ideologia sean el nuevo "motor de la historia", ni que la realidad social no tenga aspectos "materiales»: precisamente esta es una de las críticas que hace al iidealismo fenomenológico" de ciertas sociologías "comprensivas" que identifican sociedad con "mundo de la vida", o a la hermenéutica de Gadamer (Habermas, 1981a: 169215). Lo que sí dice es que los fenómenos sociales están siempre lingüisticamente mediados, y que del uso social del lenguaje se derivan unos potenciales que son muy importantes para fundamentar una teoría social con vocación crítica, y para explicar la evolución social. El esquema marxista de la base y la superestructura es, sin duda, incluso con todos los matices "dialécticos" que se quiera, demasiado simplista para dar cuenta de las complejas sociedades capitalistas actuales, pero la búsqueda de categorías nuevas no supone para Habermas un olvido del espíritu "materialista" que animaba a Marx ${ }^{11}$.

El giro lingüístico de la teoría crítica, iniciado por Habermas a finales de los sesenta, se ha ido convirtiendo con los años en lo que el propio autor ya denomina "giro pragmático". ¿Qué añade esta expresión a lo ya dicho? La complicada argumentación habermasiana en este punto se puede sintetizar como sigue: como hemos dicho, el lenguaje no sólo tiene funciones cognitivas o de transmisión de información, sino también de coordinación de la acción y de "apertura de mundom. En este sentido, al hablar hacemos uso de forma no consciente de unas estructuras generales de la comunicación lingüística. Concretamente, en cada acto de habla estándar entablamos tres pretensiones de validez con nuestro oyente: pretendemos que lo que decimos es verdadero (pretensión de verdad), que se adecúa a las normas morales que rigen este tipo de interacciones (pretensión de rectitud normativa), y que somos sinceros al decirlo (pretensión de veracidad); normalmente estas pretensiones se presuponen y se aceptan sin problemas en la vida cotidiana: nótese que Habermas no dice que las pretensiones respondan a la

11. Así lo confirma el propio Habermas en declaraciones como esta: «Desde mis primeras publicaciones he enzendido el materialismo en el sentido marxista, como un enfoque reoríco que no sólo afirma la dependencia de la superestructura con respecto a la base, del mundo real con respecro a los imperativos del proceso de acumulación, como constante ontológica, por asi decirlo, sino que a la vez explica y denuntia esa dependencia como la función latente de una formación social concreta e hisróricamente transitoria. [...] Continuo explicando el modelo selectivo de modernización capitalista, y las patologías correspondientes a un mundo racionalizado unilateralmente, en términos de un proceso de acumulación capitalista que está en buena medida desconectado de las aplicaciones orientadas hacia el valor de uson (Habermas, 1984a: 80). 
situación real, ni que la gente siempre se "crea" lo que le dicen, sino que usualmente estas pretensiones de validez no son puestas en cuestión abiertamente en la comunicación (de otro modo sería imposible la vida social). Pues bien, Habermas piensa que en ese sistema de pretensiones de validez reside un potencial de racionalidad del lenguaje - potencial que soblo en contadas ocasiones se realiza en la práctica-: precisamente cuando por cualquier razón social esas pretensiones se cuestionan públicamente en la comunicación, es cuando se hace necesario defenderlas y criticarlas con argumentos que busquen convencer a los demás; se interrumpe entonces el curso teleológico de la acción y se entra en el ámbito del discurso, en el cual, tras un proceso público de discusión - en el que por supuesto los intereses y las relaciones de poder están siempre detrás de los argumentos de cada cual-, puede alcanzarse un nuevo consenso que permita de nuevo coordinar lingüfsticamente la acción; ello es potencialmente racional porque entra en juego la fuerza de la argumentación pública, que busca un asentimiento libre de coacción, motivado únicamente por razones (aunque rara vez lo encuentre en estado "puron): en la medida en que los consensos sociales sean alcanzados más en base a tales argumentaciones que en base a relaciones de poder, imposición o manipulación, en esa medida tales consensos y las prácticas subsiguientes serán más racionales comunicativamente hablando. $\mathcal{O}$, en otras palabras, cuando la situación social permite que la discusión de pretensiones de validez pase por delante de las meras pretensiones de poder, es cuando los potenciales de racionalidad propiamente humanos se realizan en la práctica.

La racionalidad comunicativa a la que Habermas se refiere es, por tanto, una racionalidad pragmática, a diferencia de todas las demás ideas de la racionalidad que han imperado en el pensamiento occidental hasta bien entrado el siglo XX. Se trata de una racionalidad que permite coordinar y reproducir procesos sociales tan cotidianos a todos los niveles como son la socialización, la integración social o la legitimación. Es formal - no tiene contenidos sustantivos-, es procesual, y estáx "encarnada" potencialmente en las prácticas cotidianas concretas de individuos concretos en sociedades concretas. Es una racionalidad que surge y toma forma a partir de ciertas circunstancias históricas y sociales -en concreto, a partir de los procesos de modernización que en las sociedades occidentales "emancipan" cada vez más las prácticas sociales respecto de las ataduras de la tradición, la religión, la autoridad arbitraria, etc.-, pero que al mismo tiempo tiene un potencial de universalidad desde el momento que cualesquiera individuos capaces de habla y acción pueden ejercerla. Es una racionalidad, además, que rebasa la pura racionalidad instrumental o estratégica para centrarse en el modo en que los seres humanos justificamos o criticamos argumentalmente nuestros actos, creencias y afirmaciones, así como las normas, relaciones e instituciones sociales bajo las que vivimos. La "razón", tema clásico de la filosofia, da paso aś́ a una racionalidad social, cotidiana, "situada", no fundamentalista ni absolutista, y no reducida en términos instrumentalistas ni ćnicos. $\mathrm{Y}$ al mismo tiempo pasa a ser un tema sociologico a la par que filosófico: las ciencias sociales no pueden desentenderse de él, sino que son la base para desarrollar una teoría de la raciona- 
lidad ${ }^{12}$. Esta socialización de la racionalidad, que supera y disuelve las antinomias tradicionales como contextualismo-universalismo, génesis-validez o absolutismo-relativismo, es lo que se denomina "giro pragmáticon ${ }^{13}$.

\section{La teoría de la acción en Habermas: acción comunicativa y acción estratégica}

Todo lo anterior tiene una aplicación en la teoría sociológica de la acción, que se concreta en el concepto, clave para Habermas, de acción comunicativa. Para llegar a este concepto, Habermas efectúa algunas distinciones analíticas que le permitan definirlo con precisión. Veámoslas paso a paso.

1. En primer lugar, Habermas distingue entre dos tipos puros de actividad que están presentes en cualquier acción social: habla y actividad teleológica. El habla es para Habermas aquella actividad que está "orientada hacia el entendimienton, mientras que la actividad teleológica es aquella que se encuentra "orientada hacia el éxiton. Se trata, por tanto, de dos tipos de finalidades analíricamente distintas: una cosa es buscar un entendimiento intersubjetivo con otros sujetos acerca de algo en el mundo (natural, social o subjetivo), y otra muy distinta es buscar la consecución de determinados efectos o impactos sobre ese mundo, esto es, buscar el éxito práctico en nuestra acción ${ }^{14}$. Es cierto que, reduciendo esta distinción al absurdo, cabría concebir también el "habla» como una actividad teleológica, puesto que alcanzar el entendimiento con un semejante es también un determinado "impacto" en el mundo susceptible de ser conseguido o no con éxito; Habermas no niega esto: es evidente que cualquier tipo de actividad intencional que podamos concebir tiene una estructura teleológica, orientada al éxito. Pero esta reducción al absurdo no es importante para su argumento pricipal: Habermas piensa que el tipo de "éxito" consistente en lograr un entendimiento lingüístico es conceptualmente muy distinto de los demás tipos de éxitos instrumentales, puesto que introduce un tipo de racio-

12. En el fondo, y como brillantemente afirma Vilar, "estamos ante la tentativa más consistente y refinada de fundamentar filosóficamente una idea que hoy prácticamente es de sentido común: que la razón no es propiedad exclusiva de nadie y que se construye cooperativamente a través del diálogo: sea en la ciencia o en la técnica, sea en la moral, en la política o en la legislación" (Vilar, 1993).

13. Las implicaciones del "riro pragmático" son desarrolladas por Habermas sobre todo en la que sin duda es su obra más importante en los últimos diez años, Pensamiento postmetafisico (1988b).

14. En el fondo, Habermas está aquí elaborando teóricamente lo que son intuiciones elementales acerca de nuestra vida en sociedad: en primer lugar, que cuando hablamos, lo hacemos para entendernos sobre ciertas cosas - aunque evidentemente pueda haber otras intenciones más altá del entendimiento detrás de nuestros actos de habla: aquí entrarian los aspectos teleológicos de la acción-; y en segundo lugar, que cuando actuamos, lo hacemos para conseguir determinadas cosas que nos hemos propuesto. En algunos pasajes de su obra, Habermas sustituye la expresión "habla" por "actos de habla", y la expresión "actividad teleológica" por "acción", pero los significados son los mismos. 
nalidad especifica, la racionalidad comunicativa basada en pretensiones de validez que comentábamos en la sección anterior. Por tal razón, Habermas se limita a llamar "actividad orientada al exito" a toda aquella que no precisa de este tipo de racionalidad, es decir, a toda la que no pasa por un entendimiento lingǘstico intersubjetivo, y no es, por tanto, "acción orientada al entendimiento" - $\mathrm{o}$ a ese peculiar tipo de éxito que es el entendimiento.

2. Pero la distinción es aún más compleja: habla y actividad teleológica se distinguen sólo analíticamente, esto quiere decir que en el mundo real, empiri$c o$, se encuentran absolutamente entremezcladas y son inseparables. De hecho, para Habermas, cualquier acción social se compone a la vez de habla y de actividad teleológica -excepto un peculiar tipo de acción que Habermas distingue de las demás, y que llama discurso, como avanzábamos en la sección anterior: en el discurso, los cursos teleológicos de actividad quedan "suspendidos" hasta que se logra un nuevo acuerdo intersubjetivo-. Hemos pasado ya, por tanto, de una distinción entre tipos de actividad al concepto de acción social la acción social no es una acción cualquiera, sino una interacción, es decir, una accion en la cual entran en juego las acciones de otros sujetos; por tal motivo, cuando realiza análisis más concretos, Habermas habla más de plexos de acción que de acciones individualizadas: en una acción social, en una interacción, siempre entran en juego las expectativas y las prácticas de varios sujetos capaces de acción.

La acción social o interacción se caracteriza, por lo general, por ser una interacción lingüisticamente mediada - «por lo general", porque en algunos casos no ocurre así; por ejemplo, en una pelea a puñetazos, o en una persectción-. Las interacciones lingüisticamente mediadas son, por tanto, las acciones sociales sui generis para Habermas. Y, como hemos dicho, tienen siempre dos componentes: habla (si no, no serían lingüísticamente mediadas) y actividad teleológica (si no, no serían acciones).

3. Existen, evidentemente, diversos tipos de acción social — de interacción linguísticamente mediada - y varios criterios para distinguirlas. En su Teoría de la acción comunicativa, Habermas distinguía básicamente entre cuatro tipos de acción social; la estratégica, la regulada por normas, la dramatúrgica y la comunicativa. Vamos a definir brevemente cada tipo de acción, para después pasar a ver cómo se conectan con la mencionada distinción entre habla y actividad teleológica:

a) La acción estratégica es, en realidad, un subtipo -el subtipo social- de la acción teleologica - no confundir con actividad teleológica: ver punto 1 . La acción teleológica es aquella en la cual «el actor realiza un fin o hace que se produzca el estado de cosas deseado eligiendo en una situación dada los medios más congruentes y aplicándolos de manera adecuadà (Habermas, 1981a, I, p. 122). Esta acción puede ser instrumental - cuando no contamos para nada con la capacidad de acción y decisión de otros sujetos: por 
ejemplo, si actuamos sobre la naturaleza inanimada, o efectuamos operaciones técnicas-, o bien estratégica: sólo esta última es acción social. En ella, el actor intenta maximizar su utilidad haciendo un cálculo racional en el que se incluyen las expectativas de éxito y las decisiones de otros actores que también actứan estratégicamente. La acción estratégica se corresponde, por tanto, con lo que Weber llamaŕa eacción racional con arreglo a fines", o con la "elección racional" que estudian los individualistas metodológicos y la teoría de juegos. Pero, contra lo que parecen suponer estos últimos, Habermas afirma que hay otros tipos de acción social posibles, y lo que es más, que uno de ellos también es acción racional, aunque según otro tipo de racionalidad - la comunicativa.

b) La acción regulada por normas es aquella en la cual los actores se orientan por valores compartidos o normas de acción comunes, cumpliendo así una expectativa de comportamiento social. La teoría de los roles en sus diferentes versiones daría cuenta de este tipo de comportamientos. Sin embargo, para Habermas, este tipo de acción, por frecuente que pueda ser, no es conceptualmente diferente del tipo de acción teleológica: su estructura es similar, se trata de una acción "parasitaria», puesto que "al igual que en el modelo teleológico de acción, la acción es concebida primariamente como relación entre el actor y un mundon externo (ibídem: 130). Dicho de otro modo, la concepción normativa de la acción sigue dentro del paradigma de la "filosofía de la conciencian: un actor se enfrenta a un mundo exterior, aunque en este caso se trate del "mundo social" de las normas y los valores comunes; éstos se le imponen como algo objetivo, con el mismo poder coercitivo que las fuerzas naturales o las estrategias racionales de otros actores. Este tipo de acción, por tanto, no incorpora ninguna racionalidad esencialmente distinta de la teleológica o instrumental-estratégica.

c) La acción dramatúrgica es aquella en la cual los acrores se constituyen en "público" los unos para los otros, y "representan" una determinada escenificación o papel, con el conocimiento y consentimiento de ese público. Se trataría del típo de acciones estudiadas por autores como Goffman y los interaccionistas simbólicos. Sin embargo, este tipo de acciones tampoco introducen ninguna novedad esencial respecto de la acción teleológica: "Las cualidades dramatúrgicas de la acción son en cierto modo parasitarias: van montadas sobre una estructura de acción teleológica" (ibidem: 131), ya que lo que se busca es provocar instrumentalmente un cierto efecto en el público. Además, el mundo exterior sigue imponiendo sus coerciones al sujeto aislado, aunque esta vez se trate del propio «mundo de la subjetividad" del individuo, que condiciona y limita lo que éste puede representar con éxito y lo que no, con la misma objetividad y poder coactivo con que antes lo hacían la naturaleza, los agentes racionales, o las normas sociales.

d) Será, por tanto, en la acción comunicativa donde Habermas encuentra los potenciales de una racionalidad alternativa a la instrumental-estratégica 
- la racionalidad comunicativa que antes hemos definido-. La acción comunicativa es aquella en la que ulos actores buscan entenderse sobre una situación de acción para poder así coordinar de común acuerdo sus planes de acción y con ello sus acciones" (ibídem: 124). Esta es una de tantas definiciones que Habermas ha dado de la acción comunicativa, y una de las más claras y sencillas: en ella las palabras clave son "entenderse" y "cootdinary. En primer lugar, de lo que se trata es de negociar una definición de la situación a partir de las interpretaciones de cada cual, y de negociarla argumentalmente -es decir, por medio de la aceptación o rechazo de pretensiones de validez, no de pretensiones de poder, o de imposiciones dogmáticas-. Pero el entendimiento intersubjetivo así logrado debe ser el punto de partida para una coordinación de los planes de acción individuales de los actores. $O$, dicho de otro modo, y contra lo que piensan muchos, la acción comunicativa no consiste sólo en hablar, consiste también en actuar (como dijimos, toda acción se compone de habla y de actividad teleológica). A poco que lo pensemos, encontraremos que la vida cotidiana es bastante rica en acciones de este tipo, aparentemente triviales, pero que no pueden ser encuadradas en ninguno de los otros tres tipos de acción: cuando decidimos con unos amigos qué película ir a ver al cine, o a dónde ir a cenar; cuando dividimos, de común acuerdo, las tareas a realizar en un grupo de trabajo; cuando concertamos una cita, cuando transmitimos un saber o un conocimiento práctico a otros, etc., estamos utilizando el lenguaje para alcanzar un entendimiento intersubjetivo en base al cual coordinamos nuestra acción con la de otros individuos. Evidentemente, las acciones comunicativas pueden ser de un tipo mucho más complejo, menos triviales y menos cotidianas (por ejemplo, la organización y realización de acciones políticas por parte de algunos movimientos sociales, la toma de decisiones en asambleas, la investigación científica en equipo, etc.). Es obvio también que ningún tipo de acción se da nunca en estado "puro" en el mundo real. Pero de todas formas, conviene retener que lo importante en la conceptualización que Habermas hace de la acción comunicativa es:

- que el mecanismo de coordinación de la acción es el entendimiento lingüístico intersubjetivo: esta es la diferencia básica con los otros tipos de acción;

- que en ella se sale ya del paradigma de la "filosofía de la conciencia" y de la racionalidad instrumental-estratégica: no se trata de un actor que se enfrenta a un mundo que se le impone (objetivo, social o subjetivo) e intenta provocar instrumentaimente un efecto en él, sino de varios actores que negocian sus definiciones e interpretaciones de ese mundo hasta alcanzar un entendimiento que les permite actuar coordinadamente; en el que puede concebirse como caso límite de acción comunicativa, el discurso - tal como lo hemos definido en la sección anterior-, la única "coerción" existente es la del mejor argumento; 
- que es en ella donde pueden "liberarse" o "realizatse", en mayor o menor grado - no se trata nunca de una cuestión de "todo o nada" - los potenciales de la racionalidad comunicativa de que hablábamos en el apartado anterior; es decir, de una racionalidad alternativa a la instrumental-estratégica, basada en el diálogo y en la argumentación de pretensiones de validez, no en el cálculo maximizador de utilidad o de eficacia medios-fines.

4. La primera de estas tres últimas observaciones nos da ya una pista sobre qué tienen que ver estos tipos de acción social con la distinción entre habla y actividad teleológica - tipos de actividad que se encuentran en cualquier acción social- Es en Pensamiento postmetafisico donde Habermas establece más claramente esta conexión. Aquí ya sólo habla de dos tipos de acción, la estratégica y la comunicativa - hemos visto que los otros dos, la dramatúrgica y la orientada por normas, aunque sean diferentes, se estructuran sin embargo en base a la misma racionalidad que la estratégica-. Y el criterio que distingue una de otra es cuál es el mecanismo de coordinación de las acciones en cada caso. $\mathrm{O}$, dicho de otro modo, la manera como se articulan habla y actividad teleológica en cada una de ellas. En realidad, cualquier acción social, esto es, cualquier interacción, "puede entenderse como la solución del problema de cómo los planes de acción de varios actores pueden coordinarse entre sín (Habermas, 1988b: 72), reduciendo así el espacio de las múltiples concatenaciones de acción que serían en principio posibles.

En la acción estratégica y en la acción comunicativa se dan distintas formas de coordinación de la acción, es decir, habla y actividad teleológica tienen papeles distintos en cada una de ellas: se distinguten porque en la acción estratégica el lenguaje, el habla, se utiliza sólo "como medio en el que tiene lugar la transmisión de informaciones" o como "influjo que acaba induciendo a un determinado comportamienton (ibídem: 73); mientras que en la acción comunicativa ese mismo lenguaje se convierte en "fuente de integración social", dado que "la fuerza generadora de consenso del entendimiento lingüístico, es decir, las energías que el propio lenguaje posee en lo tocante a crear vínculos, se tornan eficaces para la coordinación de la acción» (ibídem). O, todavía más claro, de nuevo en palabras del propio Habermas:

La acción comunicativa se distingue, pues, de la acción estratégica porque la coordinación lograda de la acción puede hacerse derivar no de la racionalidad teleológica de las orientaciones de acción, sino de la fuerza de motivar tacionalmente que tienen los procesos de entendimiento, es decit, de una racionalidad que se manifiesta en las condiciones del acuerdo comunicativamenre alcanzado (ibídern: 132).

En un pasaje como este se resume, quizás, lo que es la aportación fundamental de Habermas a la sociología de la acción: la racionalidad comunicativa que "reside» en el lenguaje se manifiesta en un tipo específico de acción, la 
acción comunicativa, que no puede en absoluto reducirse al tipo de acción estratégica ni a la racionalidad teleológica que las «teorías de la acción racional" toman a priori como la única acción y racionalidad posibles ${ }^{15}$. Pero, al mismo tiempo, tampoco puede asimilarse a otros tipos de acción regulados por la costumbre, los valores o las normas sociales, las tradiciones, etc., dado que en éstas se da un mero seguimiento de pautas preestablecidas, sin entablar una discusión en base a la argumentación de pretensiones de validez, ni una negociación de las distintas definiciones de la situación que busque un entendimiento no coaccionado. Es en este sentido que la acción comunicativa representa un potencial de racionalización de la acción social, más amplio que el meramente estratégico, y que conecta con la idea, central en la tradición marxista, de emancipación.

\section{La teoría de la sociedad de Habermas: sistema y mundo de la vida}

A diferencia de otras escuelas sociológicas, Habermas piensa que no es suficiente una teoría de la acción para dar cuenta de la complejidad de la vida social. Es menester también abordar el problema clásico de cómo surge el "orden social" a partir de acciones aisladas. Para ello, la teoría de la acción comunicativa construye un concepto de sociedad estructurado en dos niveles: el sistema y el mundo de la vida. En esta sección veremos, ya más brevemente, en qué consiste esta original propuesta teórica de Habermas, y qué relación guarda con su teoría de la acción y de la comunicación.

En realidad la mencionada originalidad no se encuentra tanto en los conceptos de "sistema" y "mundo de la vida" como tales, que están tomados, respectivamente, de dos tradiciones sociológicas ya existentes: el funcionalismo sistémico de autores como Parsons y Luhmann, y la sociofenomenología de Schütz. Lo realmente innovador es el concebir la sociedad a la vez desde ambos puntos de vista, evitando así los reduccionismos típicos de esos dos tipos de teorías. Además, la reelaboración que hace Habermas de ese par conceptual, y sobre todo su incardinación en una teoría de la evolución social, dan a su

15. Conviene observar de nuevo que el argumento puede "reducirse al absurdo", pero que ello no tiene efectos perniciosos pata lo que Habermas está diciendo: en efecto, en un sentido trivial, cualquier acción -incluida la comunicativa - es una acción teleológica en el sentido de que trata de provocar impactos sobre el mundo. Por eso Habermas se toma tantas molestias en distinguir entre los dos componentes de toda acción - habla y actividad releologica - y en insistir en que lo que diferencia las acciones comunicativas es el modo en que rales componentes se articulan. De otro modo: también en la acción comunicativa, como en ctralquier otra, existe actividad teleológica, pero la diferencia es que los planes de acción teleológicos de los individuos se han coordinado en base a un entendimiento lingüistico. Habermas deja claro este punto en diversos pasajes de su obra, por ejemplo: "Como cualquier acción, también la acción comunicativa es acción teleológica. Pero la teleologia de los distintos planes de acción y de las acciones ejecuradas queda interrumpida aquí por el mecanismo coordinador de la acción que es el entendimiento" (Habermas, 1988b: 133; s.o.). 
teoría un poder explicativo mucho más global y la conectan con las aportaciones marxistas, hasta el punto de que, como reza el título de uno de sus libros, la teoría de la evolución de Habermas se podría considerar como una "reconstrucción del materialismo histórico" marxiano.

Empecemos por el concepto de "mundo de la vida", perfilado en su Teoría de la acción comunicativa, y perfeccionado y profundizado en obras posteriores -principalmente en Pensamiento postmetafisica-. Este concepto, según Habermas, es necesario para evitar caer en las antinomias de las teorías individualistas cuando intentan explicar el orden social a partir de acciones estratégicas aisladas. El "mundo de la vida" designa aquellos ámbitos de acción no estructurados formalmente donde los actores sociales interaccionan en base a un acervo de saber de fondo común y aproblemático, "dado por supuesto". El mundo de la vida se constituye, por tanto, en base a procesos de entendimiento lingüístico, es decir, que es de él de donde la acción comunicativa extrae sus potenciales. Una sociedad no puede ser explicada sin hacer referencia a todo este conjunto de saberes, valores, normas, etc., que constituyen el "fondo comúr" a partir del cual nace la interacción social. Los procesos de transmisión cultural de saberes y normas, de integración social y de socialización, serían imposibles de explicar sin este concepto, no pueden ser reducidos a una lógica estratégica, exigen del entendimiento lingǘístico para poder teproducirse adecuadamente.

En esta última afirmación ya se apunta una primera diferencia entre el modo en que Habermas entiende el mundo de la vida y las teorías fenomenológicas que lo utilizaron con anterioridad. Para Habermas, la concepción de la sociedad como "mundo de la vida" no puede significar una deriva idealista: por el contrario, él siempre insiste en criticar la identificación fenomenologica entre mundo de la vida y "cultura" (o construcciones o definiciones de la realidad); el mundo de la vida no está "flotando" por encima de las cabezas de los actores sociales, sino que se concreta en prácticas y en procesos que son también materiales: las instituciones y prácticas sociales de todo tipo están "ancladas" en el mundo de la vida, que es mucho más que un usaber implíciton: es una forma de vida concreta.

La segunda diferencia entre el concepto de mundo de la vida en Habermas y en las teorias fenomenológicas y hermenéuticas es de mucho mayor calado para las pretensiones de una teoría crítica de la sociedad: para Habermas, el mundo de la vida que nos viene dado no es un horizonte absolutamente irrebasable - de otro modo sería imposible explicar el cambio social acontecido en la modernidad-; aún más: es posible - y de hecho Habermas afirma que históricamente ha ocurrido- racionalizar el mundo de la vida en mayor o menor grado. Habermas está diciendo, en el fondo, que no tenemos por qué aceptar acríticamente las tradiciones y formas de vida legadas por nuestros antepasados; pero aún va más allá: podemos instaurar formas de vida cuyo mundo de la vida esté estructurado de tal manera que permita que la racionalidad comunicativa domine sobre el seguimiento acrítico de normas. Se trata, sin duda, de un proceso histórico lento, pero que según Habermas ya dio comienzo con el advenimiento de la modernidad. 
¿Qué es lo que ha ocurrido en el proceso de modernización social occidental? Básicamente, como advirtió Wéber, que dentro del mundo de la vida se han diferenciado distintas esferas de valor (cognitivas, normativas, estéticas), que a su vez se han plasmado en instituciones sociales diferentes de las de la sociedad tradicional, y que lo han hecho en base a puntos de vista universalistas. Todo esto es lo que posibilita una racionalización del mundo de la vidas esta racionalización consiste en que, al hacerse autónomos tres cipos de discurso (teórico, práctico y estètico), con sus respectivas lógicas y criterios de validez específicos, se hace posible cuestionar las legitimaciones tradicionales de tipo religioso-metafísico y alcanzar acuerdos tan sólo en base a la discusión argumental, sin necesidad de recurrir a la tradición, la autoridad, el dogma, etc.:

un mundo de la vida puede considerarse racionalizado en la medida en que permite interacciones que no vienen regidas por un consenso normativamente adscrito, sino - directa o indirectamente- por un consenso comunicativamente alcanzado. [...] la necesidad de consenso tiene que ser satisfecha cada vez más a menudo mediante un acuerdo [...] racionalmente motivado (Habermas, 1987b, I: 434-435).

Es evidente el papel que la acción comunicativa y la correspondiente racionalidad juegan en esto último. Pero a continuación Habermas echa mano de la teoría de sistemas para explicar cómo la racionalización del mundo de la vida permite otro paso en la evolución social: el que denomina diferenciación entre sistema y mundo de la vida. En efecto, conforme la racionalización avanza, la sociedad se va haciendo más compleja y diferenciada, y en los ámbitos de acción que aseguran la reproducción material de la sociedad el entendimiento lingǘstico puede ser sustituido por mecanismos impersonales de coordinación de la acción, por medios sistémicos que operan upor encima de las cabezas de los individuos", independientemente de su voluntad y su conciencia. Ello es lo que ocurre, en concreto, con el sistema económico capitalista de mercado -que se regula a través del medio "dinero"-, y con el sistema administrativo del Estado moderno - que se regula a través del medio "poder"-. $Y$ no es por castualidad: como hemos dicho, estos dos subsistemas que se diferencian del mundo de la vida son los que cumplen las funciones de reproducción material de ese mundo de la vida, y al diferenciarse descargan a éste de los enormes riesgos que supone el tener que dejar esa reproducción material a cargo de un entendimiento lingüístico que no siempre es seguro se produzca. Por tanto, la mera diferenciación de ambos subsistemas, en contra de lo que suponía la tradición marxista, no es parológica en sí misma, sino que, bien al contrario, constituye un progreso en la evolución social. El problema, para Habermas, viene por otro lado.

En efecto, el proceso de modernización específicamente capitalista - no la mera diferenciación de un subsistema económico-, con su énfasis en el valor de cambio frente al valor de uso, provoca que los subsistemas no sólo se diferencien del mundo de la vida, sino que se autonomicen e invadan los ámbitos de acción que siguen integrándose a través del entendimiento, es decir, los ámbitos que cumplen las funciones de reproducción simbólica del mundo de 
la vida (cultura, integración social, socialización), funciones éstas que, a diferencia de las de reproducción material, no pueden ser adecuadamente desempeñadas por medio de mecanismos sistémicos. El mundo de la vida se ve así distorsionado por procesos de monetarización y burocratización que provienen, respectivamente, de los subsistemas económico y político-administrativo. Estos procesos perturban las funciones de reproducción simbólica de ese mundo de la vida, generando patologías sociales de diverso tipo. A esta evolución histórica que culmina en el Estado social contemporáneo Habermas le da el nombre de colonización del mundo de la vida por el sistema, y con él pretende explicar los fenómenos que Marx, Lukács y la Escuela de Frankfurt designaban como alienación y cosificacion. Todo el argumento de Habermas se resume en un pasaje clave de su Teoría de la acción comunicativa:

La hipótesis global que de todo se obtiene para el análisis de los procesos de modernización es que el mundo de la vida, progresivamente racionalizado, queda desacoplado de los ámbitos de acción formalmente organizados y cada vez más complejos que son la economia y la administración estaral y cae bajo su dependencia. Esta dependencia, que proviene de una mediatización del mundo de la vida por los imperativos sistémicos, adopta la forma patológica de una colonización interna a medida que los desequilibrios críticos en la teproducción material [...] sólo pueden evitarse ya al precio de perturbaciones en la reproducción simbólica del mundo de la vida (Habermas, 1987b, II: 432).

La modernización occidental no es, por tanto, un proceso de por sí cosificador (Lukács, Weber), ni una "dialéctica de la Ilustración" que nos aboque al imperio de la razón instrumental (Adorno y Horkheimer), sino que es más bien un proceso selectivo ( $y$, por tanto, no necesario) de racionalización social por el cual la integración sistémica se hace dominante a costa de la integración social, esto es, a costa de la racionalidad comunicativa. Pero, al mismo tiempo, un mundo de la vida progresivamente racionalizado en términos comunicativos, aunque sufra la "colonización" del sistema, está en condiciones de oponer ciertas resistencias a la misma -encarnadas, por ejemplo, en los potenciales de protesta de los "nuevos movimientos sociales»-. La crítica de Habermas a sus "maestros" de la Escuela de Frankfurt puede, por tanto, entenderse así: no estamos condenados a vivir en una "sociedad administrada", sino que sufrimos un conflicto entre dos principios de integracion. El énfasis en la comunicación acaba posibilitando a Habermas ver una salida para la cosificación que los frankfurtianos no pudieron -o no supieron- advertir.

\section{A modo de conclusión: teoría y práctica en Habermas}

Las secciones anteriores apuntan a que, en la obra de Habermas, es sin duda la acción comunicativa la que posibilita el vínculo entre teoría y práctica que toda teoría crítica de la sociedad aspira a establecer. En las sociedades occidentales actuales, según Habermas, predomina abiertamente la comunicación sistemáticamente distorsionada por la intrusión de mecanismos sistémicos en 
ámbitos que no les son propios. La liberación del potencial de racionalidad que es inherente a la acción comunicativa podría configurar procesos de ilustración donde la comunicación no se instrumentalice con fines estratégicos, sino que sirva a la formación de una voluntad racional. Tales potenciales los ve Habermas, por ejemplo, en algunos de los llamados "nuevos movimientos sociales", así como en la capacidad — hoy desaprovechada y manipulada- de los medios de comunicación de masas para configurar una opinión pública racional o para democratizar la sociedad.

La lógica de la acción comunicativa es, por tanto, la que se opone por su propia naturaleza a la coerción social, a la cosificación de las relaciones sociales. Por eso existe en ella una cierta intención utópica, aunque no sea ésta una palabra muy del agrado de Habermas. En concreto, la utopía marxista de una descosificación total del mundo social le parece a Habermas infundada: en sociedades de una elevada complefilad y diferenciación internas, como lo son las occidentales, ciertos ámbitos de acción —el económico y el administrativodeben ser integrados sistémicamente, mediante mecanismos de autorregulación como el dinero y el poder ${ }^{16}$. En este sentido, las aspiraciones clásicas del socialismo deben, para Habermas, modificarse seriamente ${ }^{17}$, puesto que un cierto grado de cosificación y de alienación, viene a decir, resulta inevitable. El problema está en hacer retroceder las fronteras de la cosificación hasta el ámbito que les es propio o que sea imprescindible para la reproducción de la sociedad, en evitar los fenómenos de "colonización del mundo de la vida", y en poner a aquellos subsistemas autonomizados bajo el control de las legitimaciones, discursivamente formadas, de ese mundo de la vida racionafizado. Todo ello, evidentemente, supone luchas y conflictos sociales, de maneta que no se debe entender, de nuevo, la propuesta habermasiana de forma idealista: como él mismo ha dicho, ula gente no lucha por abstracciones" (Habermas, 1986: 146).

Esta conexión política de la teoría de Habermas encuentra expresión, según sus propias palabras, en un "reformismo radical", emparentado con las corrientes más izquierdistas de la socialdemocracia, con los partidos postmarxistas y postcomunistas, y con ciertos sectores de los «nuevos movimientos sociales" antiproductivistas y antiestatistas. Sin embargo, es cierto que Habermas es muy reticente a concretar políticamente sus argumentos teóricos, y mucho menos a caer en la tentación de diseñar "modelos de sociedad" alternativos o "aconsejar" estrategias de acción: nunca ha visto el papel del teórico social como el de un "compañero de viaje" ni el de una "vanguardia" de nadie; su función como teórico es la de proporcionar elementos de fundamentación para la crítica social y política de lo existente desde un punto de vista emancipatorio: esto es, fundamentar cuales son las condiciones sociales de unas formas de vida emancipadas, cuyos contenidos concretos deben ser desarrollados por los propios actores sociales. Sin

16. Nórese que ello no prejuzga para nada las formas concretas (capitalistas o no, burocráticas o no) que tomen esos subsistemas autorregulados.

17. Ver Habermas (1981b, 1985b y 1990). Puede verse también, sobre este punto, Sotelo (1995). 
embargo, cuando "desciende" de las - necesarias - abstracciones teóricas, Habermas sí suele posicionarse ante problemas y debares políticos concretos, en artículos cortos, conferencias o entrevistas ${ }^{18}$. Recientemente, además, Habermas ha dado un cierto "giro político" a su teoría, y su último libro (Habermas, 1992d) constituye un intento de elaborar una teoría política y jurídica basada en la teoría de la acción comunicativa. Por otro lado, numerosos científicos sociales en la órbita de la teoría crítica han intentado aplicar a ámbitos sociales concretos algunos desarrollos de la obra de Habermas, de tal modo que la bibliografia existente hoy día al respecto, en los diversos campos de las ciencias sociales, es inmensa ${ }^{19}$. Pero analizar tales desarrollos sería ya tema para otro artículo.

\section{Bibliografía}

ANOERSON, Perry (1983). Tras las huellas del materialismo histórico. Madtid: Siglo XXI, 1986.

BERNSTEIN, J.M. (1995). Recovering Ethical Life. Jürgen Habermas and the Future of Critical Theory. Londres: Routledge.

BERNSTEIN, Richard J. (1985). "Introducción". En R.J. BERNSTEIN (ed.) (1985).

-(ed.) (1985). Habermas y la Modernidad. Madrid: Cátedra, 1988.

CalHoun, Craig (ed.) (1992). Habermas and the Public Sphere. Cambridge, Mass.: The MIT Press.

GIODENS, Anthony (1985), "Razón sin revolución? La Theorie des kommunikativen Handelns de Habermas». En R. J. BERNSTEIN (ed.) (1985).

HABERMAS, Jürgen (1962). Historia y critica de la opinión puiblica. Barcelona: Guscavo Gili, 1981.

-(1968a). Ciencia y técnica como «ideologia». Madrid: Tecnos, 1984.

-(1968b). Conocimiento e interés. Madrid: Taurus, 1982.

-(1969) "Teoría analítica de la ciencia y dialéctica" y "Concra un estrechamiento de la razón en términos positivistas". En T.W. ADORNO y otros. La disputa del positivismo en la sociología alemana. Barcelona: Grijalbo, 1972.

-(1971). Teoría y praxis. Madrid: Tecnos, 1987.

-(1973). Problemas de legitimación en el capitalismo tardto. Buenos Aires: Amorrortu, 1975.

-(1976). La reconstruccion del materialismo historico. Madrid: Taurus, 1981.

-(1977). La logica de las Ciencias Sociales. Madrid: Tecnos, 1988.

-(1981a). Teoria de la acción comunicativa (2 vols.). Madrid: Taurus, 1987.

-(1981b). "Política conservadora, trabajo, socialismo y utopia hoy". En Ensayos politicos. Barcelona: Penínstila, 1988.

-(1983). Conciencia moral y acción comunicativa. Barcelona: Península, 1985.

-(1984a). «Perfil filosófico-politicon (entrevista con P. Anderson y P. Dews). Leviatán, 22,1985 .

-(1984b). Teoria de la acción comunicativa: complementos y estudios previos. Madrid: Cáredra, 1989.

18. Véanse, por ejemplo, las recopiladas en Habermas (1991).

19. Como uno de los ejemplos más recienres e interesantes de tal aplicación, puede verse Schlosberg (1995). 
--(1984-87). Escritos sobre moralidad y eticidad. Barcelona: Paidós-ICE, 1991.

-(1985a). El discurso filosbfico de la modernidad. Madrid: Taurus, 1989.

- (1985b). "La crisis del estado de bienestar y el agotamiento de las energías utópicas". En Ensayos politicos. Barcelona: Península, 1988.

-(1986). Autonomy and Solidarity. Interviews with J. Habermas. Londres: Verso.

-(1988a). Identidades nacionales y post-nacionales. Madrid: Tecnos, 1989.

-(1988b). Pensamiento postmetafisico. Madrid: Taurus, 1990.

-(1989). "Towards a Communication-Concept of Rational Collective Will-Formation. A Thought Experiment". Ratio Jurts, 2, 2 (julio), p. 144-154.

-(1990). La necesidad de revition de la izquierda. Madrid: Tecnos, 1991.

-(1991). The Past as Future. Lincoln: University of Nebraska Press, 1994.

-(1992a). "Ciudadanía e identidad nacional: consideraciones sobre el futuro europeo". Debats, 39 (marzo).

- (1992b). "Tres modelos de democracia. Sobre el concepto de una política deliberativa". Debats, 39 (marzo).

-(1992c). Between Facts and Norms. Contribution to a Discourse Theory of Law and Democracy. Cambridge, Mass.: The MIT Press, 1996.

-(1995). "On the Internal Relation between the Rule of Law and Democracy". European Joumal of Philosophy, 3, 1, p. 12-20.

HABERMAS, Jürgen y otros (1992), "Concluding Remarks". En C. CALHOUN (ed.) (1992).

HELD, David (1980). Introduction to Critical Theory. Horkheimer to Habermas. Berkeley: University of California Press.

HELD, David; THOMPSON, John B. (eds.) (1982). Habermas: Critical Debates. Londres: Macmillan.

HOLUB, Robert (1991). Jürgen Habermas: Critic in the Public Sphere. Nueva York: Routledge.

Honnerh, Axel (1987). "Teoría crítica". En A. Giddens; J. Turner (eds.). La teoria social hoy. Madrid: Alianza, 1990.

HonNeTH, Axel; JOAS, Hans (eds.) (1991). Communicative Action. Cambridge, Mass.: The MIT Press.

HORS'TER, Detlef (1992). Habermas: An Introduction. Philadelphia: Pennbridge Books. JAY, Martin (1973). La imaginación dialéctica. Una historia de la Escuela de Frankfurt. Madrid: Taurus.

JiMÉNEZ REDONDO, Manuel y otros (1988). Racionalidad y acción comunicativa en J. Habermas. Madrid: Fundación de Investigaciones Marxistas.

KELLNER, Douglas (1993). "Critical Theory Today: Revisiting the Classics". Theory; Culture and Society, 10, 2, p. 43-60.

LARMORE, Charles (1995). "The Foundations of Modern Democracy: Reflections on Jügen Habermas". European Journal of Philosophy, 3, I, p. 55-68.

MARDONES, José María (1990). «La recepción de la Teoría Crítica en España». Isegoria, I.

MCCARTHy, Thomas (1978). La Teorta Critica de Jürgen Habermas. Madrid: Tecnos, 1987 (epílogo original de 1987).

OUTHWAIte, William (1994). Habermas. A Critical Introduction. Cambridge: Polity Press.

RASMUSSEN, David M. (1990). Reading Habermas. Cambridge, Mass.: Basil Blackwell.

Schlosberg, David (1995). "Communicative Action in Practice: Intersubjectivity and New Social Movements". Political Studies, 43, 2, junio. 
SOTElo, Ignacio (1995). "Las ideas políticas de Habermas». Claves de razón práctica, 57 (noviembre).

THERBORN, Göran (1971). "Jürgen Habermas: un ntevo eclecticismo". Teorema, 6, 1972 .

VelasCO, Juan Carlos (1994). "Acerca del giro jurídico de la teoría crítica". Isegoría, 10.

VILAR, Gerard (1993). "Razón”. Texto policopiado. Betlaterra: Universidad Autónoma de Barcelona.

WHITE, Stephen K. (1988). The Recent Work of Jürgen Habermas. Reason, Justice and Modernity. Cambridge: Cambridge University Press.

WHITE, Stephen K. (ed.) (1995). The Cambridge Companion to Habermas. Cambridge: Cambridge University Press.

WIGGERHAUS, Rolf (1986). The Frankfurt School: Its History, Theories and Political Significance. Cambridge, Mass.: The MIT Press, 1994. 